\title{
Métodos de estimação de efeitos genéticos não-aditivos para características de peso e perímetro escrotal em bovinos de corte mestiços
}

\author{
Rachel Santos Bueno ${ }^{1 *}$, Robledo de Almeida Torres ${ }^{1}$, José Bento Sterman Ferraz ${ }^{2}$, Paulo \\ Sávio Lopes ${ }^{1}$, Joanir Pereira Eler ${ }^{2}$, Gerson Barreto Mourão ${ }^{3}$, Martinho de Almeida e Silva ${ }^{4}$, \\ Elisângela Chicaroni de Mattos ${ }^{2}$
}

\footnotetext{
1 Universidade Federal de Viçosa - UFV.

2 Faculdade de Zootecnia e Engenharia de Alimentos - FZEA/USP.

${ }^{3}$ Escola Superior de Agricultura Luiz de Queiroz - ESALQ/USP.

${ }^{4}$ Universidade Federal de Minas Gerais - UFMG.
}

RESUMO - Os objetivos neste trabalho foram investigar, em uma população de bovinos de corte mestiços a obtenção dos efeitos genéticos não-aditivos para as características peso aos 205 e 390 dias e perímetro escrotal e avaliar a consideração desses efeitos na predição dos valores genéticos dos reprodutores utilizando diferentes metodologias de estimação. No método 1 , os dados foram pré-ajustados para os efeitos não-aditivos obtidos pelo método de mínimos quadrados em modelo que considerou os efeitos fixos genéticos aditivos diretos e maternos e os não-aditivos, das heterozigoses direta e materna total, e epistasia. No método 2, os efeitos não-aditivos foram considerados covariáveis no modelo genético. Valores genéticos para os dados ajustados e não-ajustados foram preditos considerando efeitos aleatórios no modelo os efeitos aditivos direto e materno e, para peso aos 205 dias, também o efeito permanente de ambiente. Os valores genéticos dos reprodutores nas categorias analisadas, para a característica peso aos 205 dias, foram organizados em arquivos com a finalidade de verificar alterações na magnitude das predições e no ordenamento dos animais, quanto aos dois métodos de correção dos dados para os efeitos não-aditivos. Os efeitos não-aditivos não se assemelham em magnitude e sentido nos dois métodos de estimação utilizados nem para as características avaliadas. Correlações de Pearson e Spearman entre os valores genéticos foram maiores que 0,94. A utilização dos métodos de estimação avaliados não implicaria em mudanças na seleção dos animais.

Palavras-chave: avaliação multirracial, heterozigose, mestiços, perdas por recombinação

\section{Estimation methods of non-additive effects for characteristics of weight and scrotal circumference in crossbred beef cattle}

\begin{abstract}
The objective of this study was to investigate, in a population of crossbred cattle, the obtainment of the nonadditive genetic effects for the characteristics weight at 205 and 390 days and scrotal circumference, and to evaluate the consideration of these effects in the prediction of breeding values of sires using different estimation methodologies. In method 1 , the data were pre-adjusted for the non-additive effects obtained by least squares means method in a model that considered the direct additive, maternal and non-additive fixed genetic effects, the direct and total maternal heterozygosities, and epistasis. In method 2, the non-additive effects were considered covariates in genetic model. Genetic values for adjusted and non-adjusted data were predicted considering additive direct and maternal effects, and for weight at 205 days, also the permanent environmental effect, as random effects in the model. The breeding values of the categories of sires considered for the weight characteristic at 205 days were organized in files, in order to verify alterations in the magnitude of the predictions and ranking of animals in the two methods of correction data for the non-additives effects. The non-additive effects were not similar in magnitude and direction in the two estimation methods used, nor for the characteristics evaluated. Pearson and Spearman correlations between breeding values were higher than 0.94 , and the use of different methods does not imply changes in the selection of animals.
\end{abstract}

Key Words: crossbred, heterozygosity, multibreed evaluation, recombination loss

\section{Introdução}

Aumento considerável na produção de bovinos de corte por meio do melhoramento genético tem sido alcançado por seleção, cruzamento e pela combinação de ambos. Desse modo, os programas de melhoramento de animais compostos ou mestiços devem incluir os efeitos aditivos e não-aditivos nas análises genéticas. 
A avaliação genética em populações multirraciais alia as informações de todos os animais pertencentes à população em estudo, puros e mestiços, e contempla nas análises os efeitos raciais diretos e maternos e os efeitos de heterose (Williams et al., 2010). A precisão das predições do mérito genético dos animais dependerá da estimação fidedigna das médias específicas por composição racial, dos desvios dos animais em relação a estas médias e das covariâncias genéticas entre parentes puros e/ou mestiços (Cardoso \& Tempelman, 2004).

O conhecimento dos efeitos genéticos aditivos, diretos e maternos raciais, e não-aditivos, heterose e epistasia, são condições primárias e essenciais ao planejamento e à continuidade de programas de cruzamento (Abdel-Aziz, 2003). Para incrementar a eficiência de seleção em populações de animais mestiços, as características devem ser adequadamente ajustadas para os efeitos de heterose (Roso \& Fries, 2000).

Os efeitos não-aditivos podem ser estimados previamente e os dados ser submetidos à avaliação genética depois de ajustados para esses efeitos. Alternativamente, os efeitos não-aditivos podem ser incluídos como covariáveis no modelo de análise. Em ambos os procedimentos, a população multirracial é analisada como raça pura.

Procedimentos adotados para identificar e quantificar esses efeitos são baseados principalmente em funções de regressão linear simples ou múltipla (Dillard et al., 1980; Skrypzeck et al., 2000).

Os objetivos neste estudo foram analisar os efeitos genéticos não-aditivos em população de bovinos de corte mestiços e avaliar a consideração destes efeitos na predição dos valores genéticos dos reprodutores utilizando-se diferentes metodologias de estimação.

\section{Material e Métodos}

Os dados foram obtidos do projeto Montana Tropical ${ }^{\circledR}$, conduzido desde 1994, em fazendas de nove estados do País. O projeto atualmente envolve aproximadamente 33 raças (Ferraz \& Eler, 2000) e propõe o agrupamento dos animais em tipos biológicos, de acordo com suas semelhanças de tipo, função, fisiologia, aspectos de crescimento e reprodução.

Segundo Ferraz et al. (1999), os tipos biológicos ou grupos propostos são: Grupo N: animais Bos indicus, incluindo-se as raças Gir, Guzerá, Indubrasil, Nelore, Tabapuã e outros zebuínos de origem africana, como o Boran; Grupo A: bovinos de origem não-zebuína adaptados aos trópicos, por meio de seleção natural ou artificial. Este grupo inclui as raças Afrikander, Belmont Red, Bonsmara, Caracu, Romo-Sinuano, Senepol e outras; Grupo B: animais Bos taurus de origem britânica, como as raças Aberdeen Angus, Devon, Hereford, Red Angus, Red Poll, South Devon etc., com predominância das raças Red Angus e South Devon; Grupo C: animais Bos taurus de origem na Europa continental, incluindo as raças Charolesa, Gelbvieh, Limousin, Pardo-Suíça, Simental, etc.

Foram utilizados dados de 120.845 animais puros e mestiços que compõem a população multirracial e um arquivo de pedigree composto de 249.827 animais. As características estudadas foram o peso aos 205 dias (P205), peso aos 390 dias (P390) e o perímetro escrotal aos 390 dias (PE390) (Tabela 1).

Foram avaliados dois métodos de estimação dos efeitos não-aditivos na avaliação genética dos animais da população composta: método 1 - dados ajustados para os efeitos não-aditivos antes da análise genética; método 2 dados ajustados para os efeitos não-aditivos incluídos na análise genética como covariáveis no modelo.

O método 1 de estimação dos efeitos não-aditivos foi avaliado em duas etapas. Na primeira etapa, para obtenção das estimativas dos efeitos não-aditivos (heterozigoses direta e materna, e epistasia), as análises foram realizadas pelo método dos mínimos quadrados, com modelos lineares incluindo apenas os efeitos fixos para cada característica utilizando-se o PROC GLM do SAS (Statistical Analysis System, versão 8.0).

O modelo utilizado nas análises é descrito como a seguir:

$$
\begin{aligned}
& y_{i j k}=\mu+C G_{i}+C I M P_{j}+a_{A}^{d}\left(A_{i j k}^{d}-\bar{A}^{d}\right)+b_{A}^{d}\left(B_{i j k}^{d}-\bar{B}^{d}\right)+c_{A}^{d}\left(C_{i j k}^{d}-\bar{C}^{d}\right)+ \\
& +a_{A}^{m}\left(A_{i j k}^{m}-\bar{A}^{m}\right)+b_{A}^{m}\left(B_{i j k}^{m}-\bar{B}^{m}\right)+c_{A}^{m}\left(C_{i j k}^{m}-\bar{C}^{m}\right)+h_{N X A}^{d}\left(N x A_{i j k}^{d}-\overline{N x A}\right)+ \\
& +h_{N x B}^{d}\left(N x B_{i j k}^{d}-\overline{N x B}^{d}\right)+h_{N x C}^{d}\left(N x C_{i j k}^{d}-\overline{N x C}^{d}\right)+h_{A x B}^{d}\left(A x B_{i j k}^{d}-\overline{A x B}^{d}\right)+ \\
& +h_{A x C}^{d}\left(A x C_{i j k}^{d}-\overline{A x C}{ }^{d}\right)+h_{B x C}^{d}\left(B x C_{i j k}^{d}-\overline{B x C}^{d}\right)+h_{T}^{m}\left(M T_{i j k}^{m}-\overline{M T}^{h}\right)+ \\
& +r_{T}^{d}\left(R_{i j k}^{m}-\bar{R}^{h}\right)+e_{i j k}
\end{aligned}
$$

Tabela 1 - Número de observações (N), média geral, desviopadrão (DP), coeficiente de variação (CV) e número de grupos contemporâneos (NGC) obtidos para as características em estudo

\begin{tabular}{lrrrrr}
\hline Característica & N & Média & DP & CV (\%) & NGC \\
\hline P205 & 118.266 & 191,59 & 31,43 & 11,52 & 1850 \\
P390 & 58.006 & 272,68 & 50,07 & 9,46 & 1733 \\
PE390 & 25.849 & 28,28 & 3,86 & 10,52 & 792 \\
\hline
\end{tabular}

P205 - peso aos 205 dias (kg); P390 - peso aos 390 dias (kg); PE390 - perímetro escrotal aos 390 dias $(\mathrm{cm})$ 
em que: $y_{i j k}=$ valor fenotípico do animal $\mathrm{k}$ ajustado para a idade à mensuração; $\mu=$ constante inerente a cada característica; $C G_{i}=$ efeito fixo do grupo de contemporâneos i; $C I M P_{j}=$ efeito fixo de classe de idade da mãe ao parto j $(\mathrm{j}=1,2, . ., 7) ; a_{A}^{d}, a_{B}^{d}, a_{C}^{d}=$ coeficientes de regressão dos efeitos genéticos aditivos diretos associados às composições dos tipos biológicos, expressos como desvio do tipo biológico $\mathrm{N} ; a_{A}^{m}, a_{B}^{m}, a_{C}^{m}=$ coeficientes de regressão dos efeitos genéticos aditivos maternos associados às composições dos tipos biológicos, expressos como desvio do tipo biológico $\mathrm{N} ; h_{N x A}^{d}, h_{N x B}^{d}, h_{N x C}^{d}, h_{A x B}^{d}, h_{A x C}^{d}, h_{B x C}^{d}=$ coeficientes de regressão das heterozigoses diretas; $h_{T}^{m}=$ coeficiente de regressão da heterozigose materna total; $r_{T}^{d}=$ coeficiente de regressão da perda por recombinação total; $e_{i j k}=$ efeito residual.

Na segunda etapa, os dados foram ajustados para as estimativas dos efeitos não-aditivos obtidas, assim como recomendado por Mourão et al. (2008), para posterior análise de predição dos valores genéticos dos animais pelo procedimento de máxima verossimilhança restrita (REML), utilizando o programa MTDFREML (Boldman et al., 1995). O modelo genético utilizado incluiu os efeitos fixos de grupo de contemporâneo, que contemplou ano de nascimento, época de nascimento, sexo do bezerro, fazenda e grupo de manejo do animal e a classe de idade da vaca ao parto e, como aleatórios, os efeitos aditivos, direto e materno. Para P205 também foi considerado o efeito permanente de meio materno.

No método 2 os efeitos das heterozigoses direta e materna total, e de epistasia foram incluídos no modelo genético como covariáveis. Os dados, não-ajustados, foram analisados com o intuito de se obterem as soluções dos efeitos fixos, covariáveis, e dos valores genéticos preditos dos animais.

Os valores genéticos dos reprodutores para a característica peso aos 205 dias, obtidos quando os dados foram pré-ajustados para os efeitos não-aditivos e quando os efeitos não-aditivos foram considerados como covariáveis no modelo de avaliação genética, foram organizados em arquivos para obtenção das correlações de Pearson e Spearman. Para estimação das correlações entre os valores genéticos obtidas pelos dois métodos, foi utilizado o PROC CORR do SAS (Statistical Analysis System, versão 8.0).

Os touros foram classificados em três grupos de acordo com o número de filhos para as características estudadas peso aos 205 e 390 dias: touros TA - com 300 ou mais filhos mensurados; touros TM - com menos de 300 e mais de 39 filhos; e touros TB - com menos de 40 filhos. Foram avaliados 3.601 reprodutores, 2.972 garrotes, 44 touros TA, 118 touros TM e 467 touros TB.

\section{Resultados e Discussão}

As estimativas dos efeitos de heterozigose direta e materna total e da perda por recombinação não se assemelham em magnitude e sentido nos dois métodos de estimação utilizados nem para as características avaliadas (Tabela 2).

Para P205 e P390, maiores estimativas foram obtidas pelo método 2, exceto para as heterozigoses materna total, que foram ligeiramente menores. O efeito de recombinação foi negativo para ambos os pesos, 205 e 390 dias, o que indica perdas por recombinação gênica que ocorreram em detrimento dos ganhos em heterozigose, fenômeno esperado em populações de animais compostos.

Para PE390, os efeitos das heterozigoses diretas foram positivos por ambos os métodos, exceto as heterozigoses em que o grupo $\mathrm{N}$ está inserido para o método 2 . O efeito da heterose materna foi nulo pelos dois métodos e os efeitos de recombinação estimaram ganhos de $0,6 \mathrm{~cm}$ e perdas de $0,8 \mathrm{~cm}$, pelos métodos 1 e 2 , respectivamente.

Os efeitos heterozigóticos e epistáticos nos modelos de análise, adicionalmente ao efeito aditivo, afetaram, respectivamente, positiva e negativamente as características

Tabela 2 - Estimativas dos efeitos não-aditivos, heterozigoses diretas e materna, e recombinação, obtidas pelos métodos de estimação 1 (M1) e 2 (M2) para as características peso aos 205 (P205) e aos 390 dias (P390) e perímetro escrotal aos 390 dias (PE390)

\begin{tabular}{|c|c|c|c|c|c|c|}
\hline \multirow[t]{2}{*}{ Efeitos não-aditivos ${ }^{1}$} & \multicolumn{2}{|c|}{ P 205} & \multicolumn{2}{|c|}{ P390 } & \multicolumn{2}{|c|}{ PE390 } \\
\hline & M1 & M2 & M1 & M2 & M1 & M2 \\
\hline $\mathrm{N} \times \mathrm{A}$ & 1,7 & 12,3 & 3,6 & 12,8 & 0,8 & $-1,5$ \\
\hline $\mathrm{N} \times \mathrm{B}$ & 7,8 & 13,6 & 16,6 & 17,3 & 1,6 & $-0,6$ \\
\hline $\mathrm{N} \times \mathrm{C}$ & 12,8 & 18,3 & 7,4 & 19,8 & 2,5 & $-0,9$ \\
\hline$A \times B$ & 6,0 & 9,5 & 7,3 & 8,8 & 0,2 & 0,5 \\
\hline$A \times C$ & 4,3 & 13,4 & $-4,4$ & 11,3 & 0,6 & 0,4 \\
\hline $\mathrm{B} \times \mathrm{C}$ & 2,9 & 7,7 & $-4,2$ & 9,8 & 1,3 & 1,5 \\
\hline M T & 4,5 & 3,4 & 2,0 & 1,7 & $-0,1$ & 0,0 \\
\hline $\mathrm{R}$ & $-5,4$ & $-0,4$ & $-3,1$ & $-11,4$ & 0,6 & $-0,8$ \\
\hline
\end{tabular}

${ }^{1}$ Heterozigoses entre os tipos biológicos: Zebu (N), Adaptado (A), Britânico (B) e Continental (C); MT - heterose materna total; R - epistasia; expressos em 100\% 
estudadas. Demeke et al. (2003), Pimentel et al. (2006), Lopes et al. (2010a) e Lopes et al. (2010b) observaram o mesmo resultado em seus estudos.

Mourão et al. (2008) avaliaram esta mesma população quanto à contribuição dos efeitos não-aditivos e obtiveram estimativas de heterose materna próximas às deste estudo, de 4,51 e 1,72 kg para as características peso aos 205 e 390 dias, respectivamente. As estimativas de heterozigose diretas foram menores, exceto para $\mathrm{A} \times \mathrm{B}$ para peso aos 205 dias e B $\times$ C para peso aos 390 dias. O modelo utilizado nestas análises não incluía o efeito de epistasia, o que pode ter inflacionado as estimativas.

Teixeira (2004) encontrou efeitos heteróticos significativos e positivos para a característica perímetro escrotal, promovendo aumento nos animais F1, Nelore $\times$ Angus e Nelore $\times$ Hereford, de 1,52 e 0,45 cm, respectivamente. No entanto, no mesmo estudo, reportou efeitos epistáticos individuais negativos em animais Nelore $\times$ Hereford, de $-2,23 \mathrm{~cm}$, observando que as perdas por recombinação na geração F2 superaram os ganhos obtidos pelos efeitos heteróticos na geração F1.

Vários trabalhos (Roso et al., 2005; Teixeira et al., 2005; Pimentel et al., 2006; Mourão et al., 2007; Bocchi et al., 2008, et al., 2010a; Lopes et al., 2010b) têm gerado componentes de efeitos genéticos aditivos e não-aditivos para populações de animais mestiços. Os resultados são variáveis e dependem das raças e dos grupos genéticos envolvidos nos cruzamentos e da contribuição das mesmas.

A estimação dos efeitos não-aditivos e o ajuste dos dados para esses efeitos visam diminuir a variabilidade decorrente da heterose, sem interferir na predição dos efeitos aditivos importantes na seleção e no melhoramento genético de populações multirraciais. O pré-ajuste dos dados para posterior análise genética foi utilizado por Mourão et al. (2007) e Bocchi et al. (2008) para a mesma população em estudo. A heterose estimada pela inclusão dos efeitos como covariáveis no modelo é um procedimento também utilizado em estudo genético de populações multirraciais (Teixeira et al., 2005; Vergara et al., 2009).

Lopes et al. (2009) estudaram informações de tourinhos das raças Aberdeen Angus, Nelore e seus cruzamentos quanto à característica perímetro escrotal e notaram a necessidade de se corrigirem estas medidas para os efeitos de grupo genético, heterozigose individual, peso corporal e idade ao sobreano para que a característica possa ser utilizada como critério de seleção para precocidade sexual.

Os modelos utilizados para analisar dados de animais mestiços devem contemplar os três principais parâmetros genéticos: efeitos aditivos (puros), efeitos de dominância (heterozigose racial) e efeitos epistáticos. No entanto, as estimativas dos efeitos genéticos não-aditivos de dominância (heterozigose racial) e epistasia obtidas comumente pelos métodos dos mínimos quadrados são pouco consistentes e de difícil interpretação prática. Roso et al. (2005) reportaram problemas de multicolinearidade quando efeitos epistáticos foram adicionados ao modelo aditivo-dominante.

Quando existem fortes relações lineares entre as variáveis independentes, a simples estimativa dos coeficientes de regressão por mínimos quadrados tende a ser instável, geralmente com grande erro-padrão, o que pode levar a inferências errôneas (Bergmann \& Hohenbolken, 1995).

Um procedimento para contornar os problemas causados pela multicolinearidade é o uso de métodos nãoviesados de estimação dos coeficientes de regressão (Pimentel et al., 2006), sendo a regressão de cumeeira um desses métodos.

Lopes et al. (2010a) estudaram uma população bovina multirracial Aberdeen Angus $\times$ Nelore para avaliar a inclusão dos efeitos não-aditivos no modelo de análise dos registros de desempenho pós-desmama utilizando diferentes modelos e três métodos de análise diferentes: método dos quadrados mínimos (MQM), regressão de cumeeira (RC) e máxima verossimilhança restrita (REML), e constataram que o método da regressão de cumeeira produziu estimativas dos coeficientes com magnitudes e sinais explicados biologicamente.

A fim de averiguar a implicação de se considerarem os efeitos não-aditivos como pré-ajuste dos dados, ou como covariáveis no modelo na avaliação genética, os valores genéticos preditos dos reprodutores para a característica P205 foram comparados quanto à alteração na magnitude e/ ou ao ordenamento dos mesmos nas diferentes categorias analisadas.

A média dos valores genéticos para as categorias de touros e garrotes foi menor quando obtida pelo método 2 . A redução foi de 44; 54 e 21\% para os touros TA, TM e TB e de $21 \%$ para os garrotes. No entanto, o desvio-padrão e a amplitude dos valores genéticos preditos dos reprodutores, touros e tourinhos praticamente não se alteraram quando foram utilizados os dois métodos de correção dos dados para os efeitos não-aditivos.

Pequena diferença foi constatada entre as correlações estimadas para os três grupos de touros. As correlações entre os dois procedimentos utilizados para correção dos dados diminuíram à medida que reduziu o número de filhos avaliados de cada touro, observado nos grupos TA, TM e TB.

Os valores acima de 0,94 para as correlações de Pearson e Spearman (Tabela 3) entre os valores genéticos preditos dos reprodutores, para peso aos 205 dias, indicam que o ordenamento dos reprodutores não foi alterado pelo método 
Tabela 3 - Estimativas de médias, desvios-padrão (DP), valores máximos e mínimos, correlações de Pearson e Spearman dos valores genéticos dos touros, classificados de acordo com o número de filhos mensurados, e dos garrotes, obtidas a partir métodos de estimação 1 (M1) e 2 (M2), para peso aos 205 dias

\begin{tabular}{|c|c|c|c|c|c|c|c|c|c|c|}
\hline & \multicolumn{2}{|c|}{$\begin{array}{c}\text { T A } \\
\text { Touros }=300 \text { filhos } \\
(\mathrm{N}=44)\end{array}$} & \multicolumn{2}{|c|}{$\begin{array}{c}\text { T M } \\
\text { Touros com } 40<\text { filhos }<300 \\
(\mathrm{~N}=118)\end{array}$} & \multicolumn{2}{|c|}{$\begin{array}{c}\text { TB } \\
\begin{array}{c}\text { Touros }<40 \text { filhos } \\
(\mathrm{N}=467)\end{array} \\
\end{array}$} & \multicolumn{2}{|c|}{$\begin{array}{c}\text { Garrotes } \\
100 \% \\
(\mathrm{~N}=2.972) \\
\end{array}$} & \multicolumn{2}{|c|}{$\begin{array}{c}\text { Garrotes } \\
20 \% \text { melhores pelo } \mathrm{M} 1 \\
(\mathrm{~N}=593) \\
\end{array}$} \\
\hline & M1 & M2 & M1 & M2 & M1 & M2 & M1 & M2 & M1 & M2 \\
\hline Média (kg) & 4,53 & 2,53 & 2,71 & 1,25 & 1,84 & 1,46 & 3,91 & 3,08 & 7,50 & 6,71 \\
\hline DP (kg) & 3,97 & 4,06 & 4,46 & 4,53 & 4,27 & 4,23 & 2,72 & 2,77 & 1,18 & 1,26 \\
\hline Mínimo (kg) & $-3,87$ & $-5,64$ & $-11,60$ & $-9,44$ & $-12,44$ & $-14,12$ & $-6,50$ & $-7,40$ & 6,14 & 4,31 \\
\hline Máximo (kg) & 12,49 & 10,47 & 13,20 & 11,77 & 15,14 & 13,27 & 15,11 & 14,07 & 15,11 & 14,07 \\
\hline M1 & 1,00 & & 1,00 & Correlações & de Pears & & 1,00 & & 1,00 & \\
\hline \multicolumn{11}{|c|}{ Correlações de Spearman } \\
\hline $\begin{array}{l}\text { M1 } \\
\text { M2 }\end{array}$ & $\begin{array}{l}1,00 \\
0,99\end{array}$ & 1,00 & $\begin{array}{l}1,00 \\
0,97\end{array}$ & 1,00 & $\begin{array}{l}1,00 \\
0,94\end{array}$ & 1,00 & $\begin{array}{l}1,00 \\
0,99\end{array}$ & 1,00 & $\begin{array}{l}1,00 \\
0,94\end{array}$ & 1,00 \\
\hline
\end{tabular}

de correção dos dados para os efeitos não-aditivos. Assim, a utilização dos métodos não implicaria na seleção de diferentes animais como reprodutores.

\section{Conclusões}

Os efeitos não-aditivos devem ser considerados na avaliação genética de populações de animais mestiços. Do mesmo modo, estudos e particularidades da população precisam ser investigados quanto aos efeitos não-aditivos e ao método de inclusão dos mesmos na análise genética. A estimação dos efeitos não-aditivos pelos métodos avaliados não alteraria a seleção dos melhores animais como reprodutores na população em estudo.

\section{Agradecimentos}

À Agropecuária CFM Ltda, pelo fornecimento dos dados; e ao CNPq, pelo apoio financeiro.

\section{Referências}

ABDEL-AZIZ, M.; SCHOEMAN, S.J.; JORDAAN, G.F. Estimation of additive, maternal and non-additive genetic effects of preweaning growth traits in a multibreed beef cattle project. Animal Science Journal, v.74, n.3, p.169-179, 2003.

BERGMANN, J.A.G.; HOHENBOKEN, W.D. Alternatives to least squares in multiple linear regression to predict production traits. Journal of Animal Breeding and Genetics, v.112, p.1-16, 1995.

BOCCHI, A.L.; OLIVEIRA, H.N.O.; FERRAZ, J.B.S. et al. Avaliação genética multirracial para ganho de peso pré-desmama em bovinos de uma população composta. Revista Brasileira de Zootecnia, v.37, n.7, p.1207-1215, 2008.

BOLDMAN, K.G.; KRIESE, L.A.; VAN VLECK, L.D. et al. A manual for use of MTDFREML: a set of programs to obtain estimates of variances and covariances (DRAFT). Lincoln: Department of Agriculture/Agriculture Research Service, 1995. $125 p$.

DEMEKE, S.; NESER, F.W.C.; SCHOEMAN, S.J. Early growth performance of Bos taurus $\times$ Bos indicus cattle crosses in Ethiopia: Estimation of individual crossbreeding effects. Journal of Animal Breeding and Genetics, v.120, p.245-257, 2003.

DILLARD, E.U.; RODRIGUEZ, O.; ROBISON, O.W. Estimation of additive and nonadditive direct and maternal genetic effects from crossbreeding beef cattle. Journal of Animal Science, v.50, p.653-663, 1980.

CARDOSO, F.F.; TEMPELMAN, R.J. Hierarchical Bayes multiplebreed inference with an application to genetic evaluation of a Nelore-Hereford population. Journal of Animal Science, v.82, n.6, p.1589-1601, 2004.

FERRAZ, J.B.S.; ELER, J.P. Desenvolvimento de bovinos de corte compostos no Brasil: o desafio do projeto Montana Tropical. In: SIMPÓSIO NACIONAL DE MELHORAMENTO ANIMAL, 3., 2000, Belo Horizonte. Anais... Belo Horizonte: Sociedade Brasileira de Melhoramento Animal [2000] (CD-ROM).

FERRAZ, J.B.S.; ELER, J.P.; GOLDEN, B.L. Análise genética do composto Montana Tropical. Revista Brasileira de Reprodução Animal, v.23, p.111-113, 1999.

LOPES, J.S.; RORATO, P.R.N.; WEBER, T. et al. Parâmetros genéticos e tendências genética e fenotípica para características de crescimento em uma população da raça Brangus. Revista Brasileira de Zootecnia, v.38, n.4, p.662-669, 2009.

LOPES, J.S.; RORATO, P.R.N.; WEBER, T. et al. Avaliação do desempenho na pós-desmama para uma população bovina multirracial Aberdeen Angus x Nelore utilizando-se diferentes modelos genéticos. Arquivo Brasileiro de Medicina Veterinária e Zootecnia, v.62, n.6, p.1439-1447, 2010a.

LOPES, J.S.; RORATO, P.R.N.; WEBER, T. et al. Pre-weaning performance evaluation of a multibreed Aberdeen Angus $x$ Nellore population using different genetic models. Revista Brasileira de Zootecnia, v.39, n.11, p.2418-2425, 2010b

MOURÃO, G.B.; FERRAZ, J.B.S.; ELER, J.P. et al. Genetic parameters for growth traits of a Brazilian Bos taurus x Bos indicus beef composite. Genetics and Molecular Research, v.6, p.1190-1200, 2007.

MOURÃO, G.B.; FERRAZ, J.B.S.; ELER, J.P. et al. Non additive genetic effects on weights and performance of a Brazilian Bos taurus $\mathrm{x}$ Bos indicus beef composites indicus composite. Genetics and Molecular Research, v.7, p.1156-1163, 2008. 
PIMENTEL, E.D.C.G.; QUEIROZ, S.A.D.; CARVALHEIRO, R. et al. Estimativas de efeitos genéticos em bezerros cruzados por diferentes modelos e métodos de estimação. Revista Brasileira de Zootecnia, v.35, n.3, p.1020-1027, 2006.

ROSO, V.M.; FRIES, L.A. Avaliação das heteroses materna e individual sobre o ganho de peso do nascimento ao desmame em bovinos Angus x Nelore. Revista Brasileira de Zootecnia, v.29, n.3, p.732-737, 2000.

ROSO, V.M.; SCHENKEL, F.S.; MILLER, S.P. et al. Estimation of genetic effects in the presence of multicollinearity in multibreed beef cattle evaluation. Journal of Animal Science, v.83, n.8, p.1788-1800, 2005.

SKRYPZECK, H.; SCHOEMAN, S.J.; JORDAAN, G.F. et al. Estimates of crossbreeding parameters in a multibreed beef cattle crossbreeding project. South African Journal of Animal Science, v.30, n.3, p.193-203, 2000.

TEIXEIRA, R.A. Efeitos genéticos aditivos e não aditivos que influenciam características reprodutivas e de crescimento em bovinos de corte. 2004. 83f. Tese (Doutorado em Zootecnia) Faculdade de Ciências Agrárias e Veterinárias, Universidade Estadual Paulista, Jaboticabal.

TEIXEIRA, R.A.; ALBURQUERQUE, L.G. Heteroses materna e individual para o ganho de peso pré-desmama em bovinos Nelore $x$ Hereford e Nelore x Angus. Arquivo Brasileiro de Medicina Veterinária e Zootecnia, v.57, n.4, p.518-523, 2005.

WILLIAMS, J.L.; AGUILAR, I.; REKAYA, R. et al. Estimation of breed and heterosis effects for growth and carcass traits in cattle using published crossbreeding studies. Journal of Animal Science, v.88, p.460-466, 2010 